\title{
Thermoactuated Diffusion Control in Soft Matter Nanofluidic Devices
}

\author{
Martin Markström, Ludvig Lizana, Owe Orwar and Aldo Jesorka* \\ Department of Chemical and Biological Engineering, Chalmers University of Technology, SE- \\ 41296 Göteborg, Sweden
}

\section{Supporting Information}

\section{$\underline{\text { FCS Data Treatment }}$}

The data was analyzed with the ISS Vista FCS LE 3.6_22 (ISS Inc., USA) software. A model assuming a 3D Gaussian beam profile in presence of a triplet state was fitted to the auto-correlation function:

$$
G(\tau)=\frac{1}{\langle N\rangle}\left(1+A \exp \left(\frac{-\tau}{\tau_{t}}\right)\right) \frac{1}{\left(1+\frac{\tau}{\tau_{d}}\right) \sqrt{1+\frac{\tau}{s^{2} \tau_{d}}}}
$$

where $\tau_{d}=\frac{w_{0}^{2}}{4 D}, s=\frac{z_{0}}{w_{0}}$, and $A=\frac{F}{1-F} \cdot<\mathrm{N}>$ is the average number of molecules in the focal volume, $\mathrm{F}$ is the fraction of molecules residing in the dark state at any time, $\mathrm{z}_{0}$ and $\mathrm{w}_{0}$ is the beam waist and beam height, respectively. $\mathrm{D}$ is the diffusion coefficient of the fluorescent molecule, $\tau_{\mathrm{d}}$ is the time a molecule stays in the confocal volume, and $\tau_{\mathrm{t}}$ is the lifetime of the triplet state. All samples were measured four times and the model was globally fitted using a Levenberg-Marquardt algorithm. 


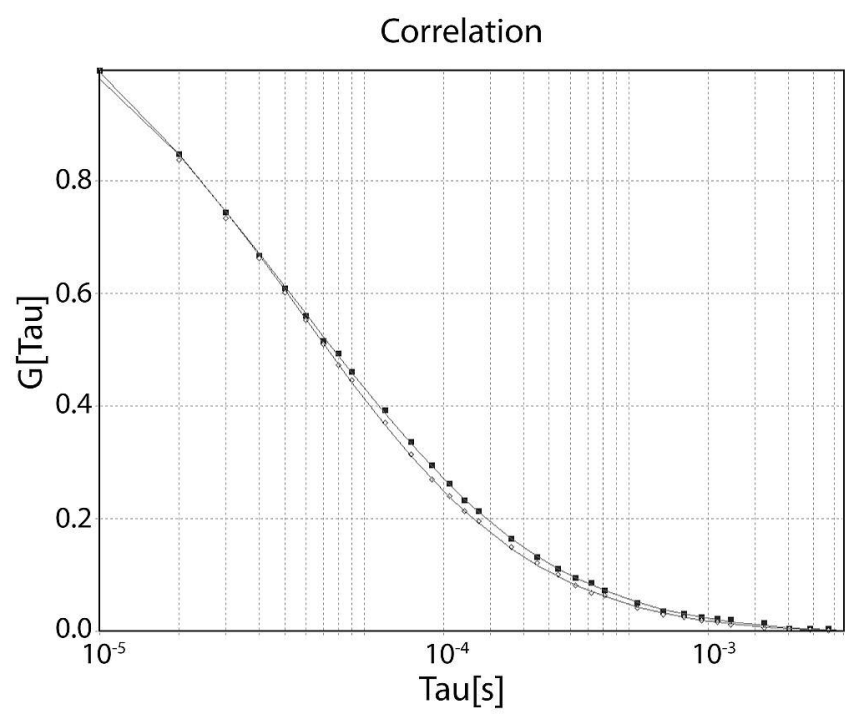

Figure S1: Measured (points) and calculated (lines) autocorrelation for Alexa Fluor 488 in buffer (light circles) and PBD solution (dark squares) Diffusion times are: $84 \mu \mathrm{s}$ and $65 \mu \mathrm{s}$ in $20 \mathrm{mg} / \mathrm{ml}$ (PBD) and $0 \mathrm{mg} / \mathrm{ml}$ PNIPAAm (buffer), respectively.

Supplementary Model Data

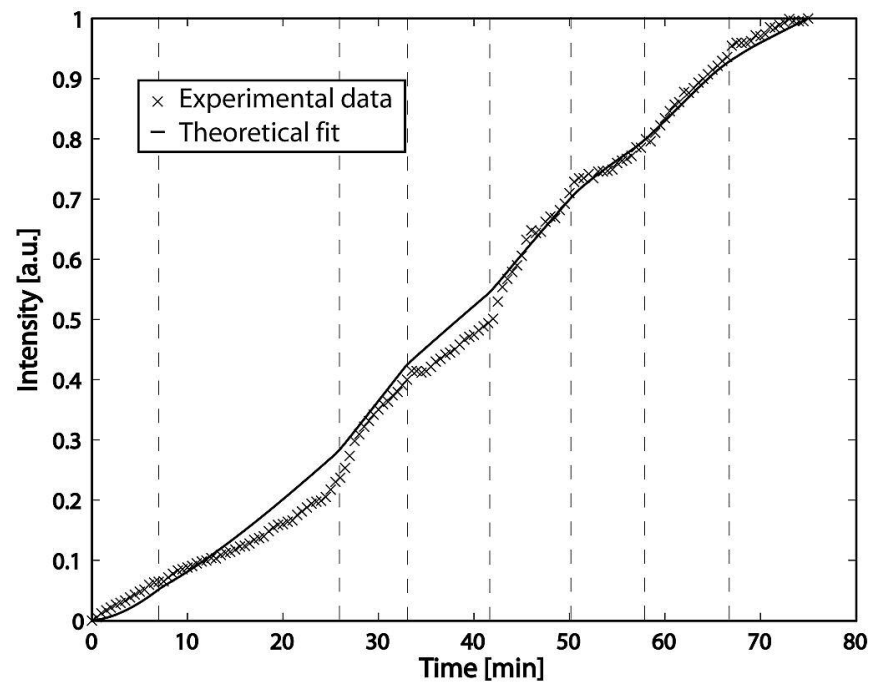

Figure S2: Average fluorescence intensity in vesicle 3 in a NVN with a PNIPAAm valve. In the open state, the diffusion coefficient in the model (Eq. 1) was multiplied with a factor 1.35 (temperature effect) and the volume of the valve vesicle was decreased by $5 \%$. 
In the closed state, the polymer effect is not considered. Vertical dashed lines denote state changes of the valve, starting in the open state.
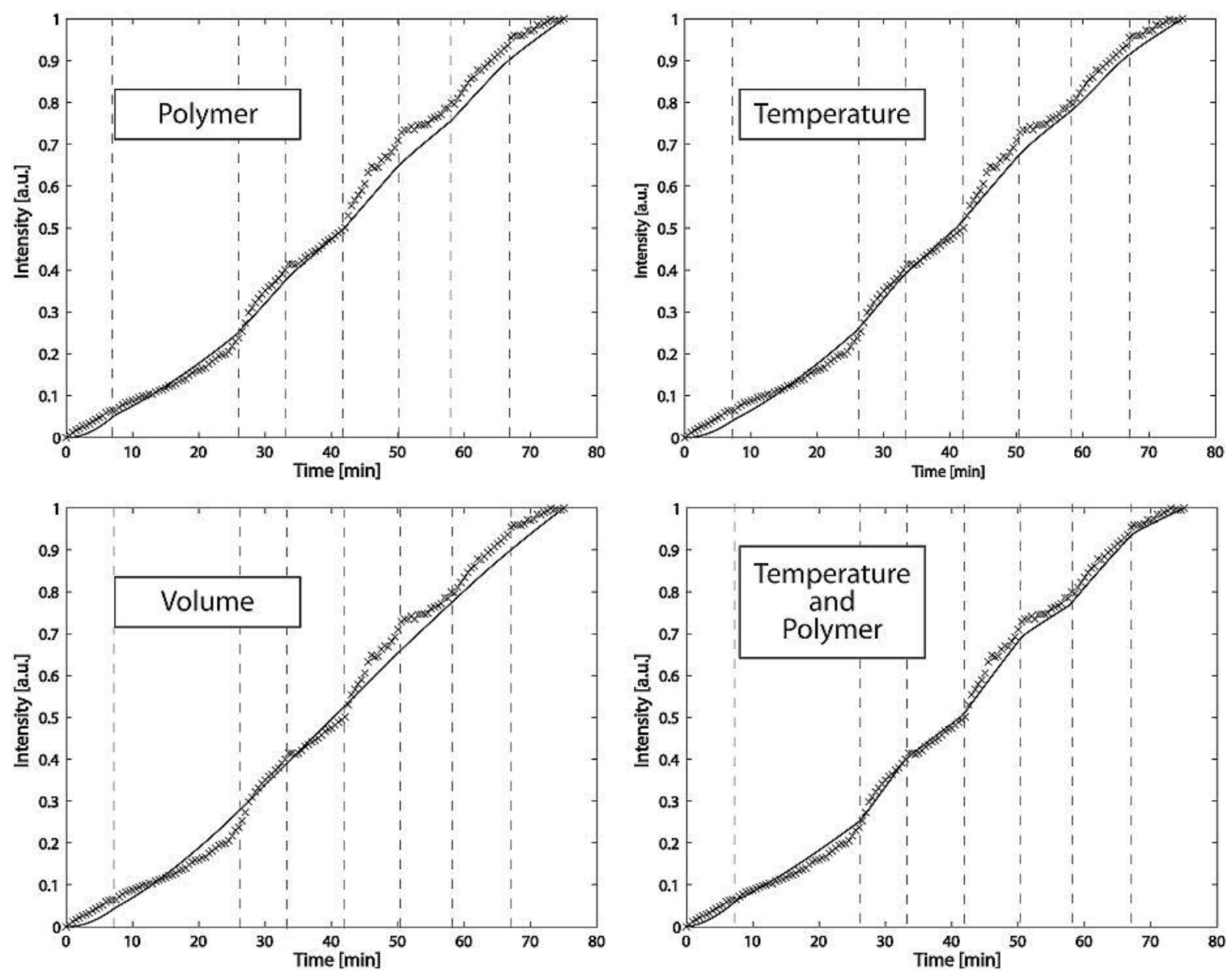

Figure S3: Average fluorescence intensity in vesicle 3 in a NVN with a PNIPAAm valve and fitted data for different model components. The texts in the panels indicate the component(s) that has/have been changed in the model (Eq. 1) between the closed and open states. In other respects, the model parameters are the same as for the fit in figure $4 \mathrm{~A}$. 